\title{
Overview of research on Surface Mechanical Attrition Treatment (SMAT)
}

\author{
Shreyas.P ${ }^{1}$, Trishul.M.A ${ }^{2}$ \\ Assistant Professor, Mechanical Engineering Department, CMRIT, Bengaluru, India ${ }^{1}$ \\ Assistant Professor, Mechanical Engineering Department, PMCE, Hosur, India ${ }^{2}$
}

\begin{abstract}
This paper is an effort to put forth a cumulative presentation of the research being carried out on Surface Mechanical Attrition Treatment (SMAT). SMAT is in some ways similar to Shot-Penning, but differs from it in Ball Size and also the angle of attack. Ball sizes in Shot-penning is in the range of $200 \mu \mathrm{m}-1 \mathrm{~mm}$, whereas in SMAT it varies from $300 \mu \mathrm{m}-10 \mathrm{~mm}^{[1]}$ so the ball size is greater in SMAT and the angle of attack or impact in Shot-Penning is $90^{\circ}$, but in SMAT the balls impact the specimen at random angles. This paper will discuss all the research that has been carried out on SMAT, like the effect of it on hardness, corrosion, phase transformation, etc. It was observed that Corrosion resistance depleted after SMAT, and the hardness of the treated surface increased but gradually decreased with increasing depth from the treated surface, the significant observation is the reduction in the grain size from a few $\mu \mathrm{m}$ to $\mathrm{nm}$. The outcome of all the research work has been explained in detail in this paper.
\end{abstract}

Keywords: Surface Mechanical Attrition Treatment (SMAT), Nancrystalization, Potentiodynamic Polarization, Phase Transformation.

\section{INTRODUCTION}

Surface Mechanical Attrition Treatment (SMAT) is a characterized using XRD, SEM and TEM observations. A method of surface treatment which was first introduced by grain refinement mechanism induced by plastic Jian $\mathrm{Lu}$ and $\mathrm{K}$. Lu in $1999^{[2]}$. In this method of treatment deformation was proposed. According to this proposition, the surface of the treated material undergoes plastic the grain refinement was caused by the formation of Dense deformation at a very high rate and there by due to the Dislocation Walls (DDWs) and Dislocation Tangles (DTs) formation of mechanical twins the grain size of the treated in the original grains, and in the refined grains under surface is converted to nano-size.

In this paper all the recent developments that have been further straining, DDWs and DTs were transformed into sub-boundaries. Then these subboundaries evolved into the field of SMAT has been indicated that high strains and high strain rates are comprehensively covered. Over the years, researchers have necessary for formation of nanocrystallites during plastic taken a huge interest in SMAT because it's a novel low deformation of the surface of the sample by SMAT.

cost method of changing the property of the material. It can be noticed from many researches that the property of a Another paper titled "Effect of surface mechanical attrition material can be altered just by changing its surface treatment (SMAT) on microhardness, surface roughness charachteristics. This is mainly because any defect usually and wettability of AISI $316 \mathrm{~L} "\left[{ }^{[4]}\right.$ authored by B.Arifvianto, starts from the surface of the material. So by strengthening Suyitno, M. Mahardika, P. Dewo, P.T. Iswanto, U.A. the surface a lot of defects can be overcome. A lot of research work on SMAT has been focussed on the improving the surface properties of a material improves Salim focuses mainly on the effects of SMAT on surface properties of $316 \mathrm{~L}$ SS. The author stress on the fact that characterization of the treated surface using Scanning the overall behavior of the material. This process is not Electron Microscopy (SEM), Transmission Electron only effective but also cheaper compared to changing the Microscopy (TEM) and X-Ray Diffraction (XRD). The structure of the entire material ${ }^{[5]}$. SMAT is performed with effect of SMAT on the hardness and the corrosion varying parameters by the authors, like, time duration of resistance of the material are also the major areas of the process, diameter of the ball and also the speed of the discussion in the papers related to SMAT.

\section{JOURNAL PAPERS}

In a paper titled "An investigation of surface nanocrystallization mechanism in Fe induced by surface mechanical attrition treatment" ${ }^{[3]}$ authored by N.R. Tao, Z.B. Wang, W.P. Tong, M.L. Sui, J. Lu, K. Lu it has been explained how nanostructured surface layer was achieved on a pure Fe Plate through SMAT. Microstructure features at various depths of the sample were systematically

motor (frequency).

The authors have concluded that the surface microhardness increases with increasing time period, ball diameter and frequency as well. Surface Roughness of the material before and after SMAT was determined using a contact profilometer. Due to the impact of the balls on the steel surface, craters were created on the surface and hence increased the surface roughness of the material by about 93-95\%. Even surface roughness is directly proportional to all the three process parameters. 
Microhardness test was also carried out on the treated Potentiodynamic polarization was used to determine the surface. Microhardness was selected instead of macro- effect of SMAT on the corrosion behaviour of the hardness test because of the depth of nano-crystalization specimen. The test was done on the As received, SMATed from the treated surface. The depth of nanocrystalization and SMATed annealed specimen. The test is carried out in from the treated surface is only about $100-200 \mathrm{~nm}$. So, in a standard three-electrode cell. Where a Saturated Calomel order to accurately measure the surface hardness of the Electrode (SCE) is the reference electrode, a platinum treated surface microhardness was selected. The results of plate is used as a counter electrode. The sample is placed the microhardness test clearly depicted that the specimen in an electrolyte medium which is $0.1 \mathrm{~mol} / \mathrm{L} \mathrm{NaOH}$ which was SMATed for the longest duration of time had Solution. The potential applied is $\quad-600 \mathrm{mV}$ at a rate the highest hardness value and the hardness values of $0.3 \mathrm{mV} / \mathrm{s}$.

gradually decreased as the time duration of SMAT decreased. This can be seen in fig. 2.1 below.

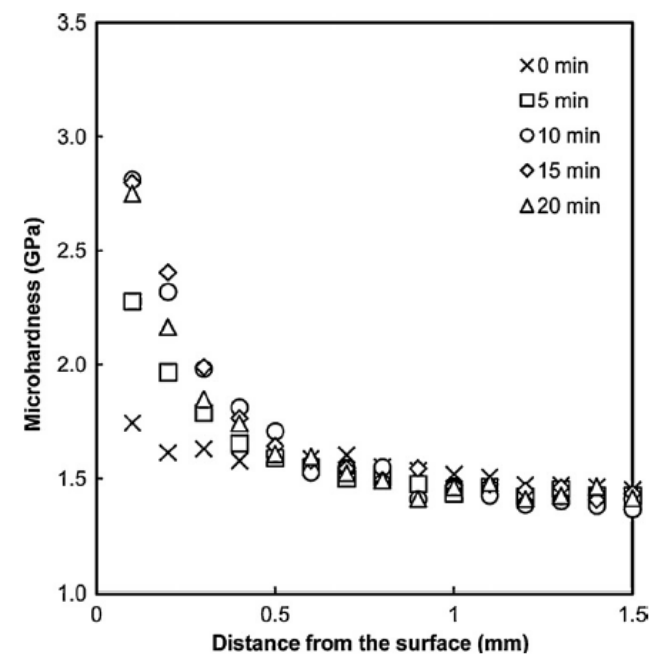

Fig. 2.1 Effect of SMAT duration on microhardness ${ }^{[4]}$. The surface wettability was quantified through a sessile drop test to obtain the droplet contact angle on each sample. The treated samples were also cleaned up using $70 \%$ ethanol, rinsed in distilled water, and dried before the measurement. A distilled water droplet was deposited three times at five different locations on the surface of each sample. The static droplet on sample's surface was recorded using a high speed camera by which the droplet contact angle analysis was then carried out ${ }^{[4]}$. The SMAT slightly enhances the surface wettability as indicated by a little reduction of droplet contact angles in all the treated samples. The droplet contact angle of the control surface is $\pm 88.6^{\circ}$; indicating its less hydrophilic property due to the polishing treatment ${ }^{[4]}$.

The effect of SMAT on the corrosion behaviour of 316L SS has been investigated by HAO YUN - Wei, et. al. and the results have been discussed in the paper titled, "Effect of SMAT on Corrosion behaviour of $316 \mathrm{SS}^{\text {"[6] }}$. The average grain size was determined by XRD this also helped in the identification of the phase transformation of the treated specimen after SMAT. It was found that the average grain size had reduced from $10 \mu \mathrm{m}$ to $19 \mathrm{~nm}$ after SMAT. Hydrogen embrittlement technique was used for determining brittleness. In hydrogen embrittlement technique, the sample is first electrolysed in a solution of $10 \mathrm{~g} \mathrm{Na}_{2} \mathrm{SO}_{4}$ and $100 \mathrm{ml}$ refined water at $0^{\circ} \mathrm{C}$ for 30 minutes with the applied potential of $500 \mathrm{mV}$. Then the specimen is acted upon by an induced shearing force which produces a cleavage plane which was later characterized using SEM. It was determined from these tests that the brittleness had increased after SMAT.



Fig 2.2 Polarization Curves of SMATed and As Received Specimen.

It can be seen from the polarization curve in fig. 2.2 that the break down potential (the part of the curve where the potential levels off to a constant value) of the As Received specimen is much higher than that of the SMATed specimen. Break down potential is nothing but the potential at which the passive protective layer that is formed on the surface of the specimen breaks down. This layer is the one that protects the metal from corrosion so when this layer breaks down it is safe to assume that the corrosion resistance of the material is also broken.

It was also determined from further investigation that the passive layer broke at a much higher potential in case of a SMATed and annealed (for 2 hours) specimen when compared to the just SMATed Specimen but was still lower than that of the As Received specimen. This can be explained by the theory that annealing produces a well defined nano sized grain boundaries which creates an interfacial bonding between the anocrystalline surface and the substrate. One of the other reasons is that during annealing Chromium diffuses from the matrix to the surface of the metal and hence creating a more resistive chromium rich passive layer which breaks at a much higher potential than just the SMATed specimen.

The disruption in the stacking of the layer in a crystalline solid is called as the stacking fault. This is caused because of the repulsive and attractive forces within the crystalline lattice. These stacking faults contain energy called as the stacking fault energy. The paper titled "Development of nano structures in metallic materials with Low Stacking Fault Energies during SMAT"[7]. The materials used in this study are Inconel 600 alooy and 304 SS. Before testing both the specimen were annealed to negate an effect of machining process and also to obtain a homogeneous coarse grains. XRD and TEM were used to characterize the grain size and structure of the SMATed specimen. 
Cross-sectional TEM observations in the SMAT Inconel 600 sample showed various types of microstructures in the surface layer, including randomly-oriented equiaxed nanocrystallites, mechanical microtwins and planar dislocation arrays with the depth increasing Note that the size of nanocrystallites increases as the depth increases in the top layer of $12 \mathrm{~mm}$ thick. Adjacent to the nanostructured surface layer, the prominent feature of microstructure is mechanical microtwins instead of submicro- and micrometer-sized subgrains or dislocation cells as observed in SMAT Fe and Al-based alloy with high SFEs. Planar dislocation arrays are found in plastic deformation regime adjacent to the strain-free matrix. Such microstructures differ from that of the SMAT Fe and Albased alloy samples in which the grain (or dislocation cell) size gradually increases from nanometer to micrometer with increasing depth.

Equiaxed randomly-oriented nanocrystallites were obtained in the surface layer of the SMAT AISI 304 sample In the regime adjacent to the strain-free matrix, the microstructure of the SMAT AISI 304 sample is characterized by planar dislocation arrays and stacking faults. The planar dislocation arrays in two directions intersect with each other with an angle of about 70.5_ (the angle between two $\{111\}$ crystallographic planes), forming dislocation grids. The spacing between dislocation arrays depends upon the orientation of original grains, ranging from $200 \mathrm{~nm}$ to $1 \mathrm{~mm}$. As strain increases, plastic deformation is accommodated by mechanical twins. Compared with the Inconel 600 sample, the prominent feature is that twin-twin intersections were observed in the AISI 304 sample.

\section{RESULTS}

It can be easily derived from all of the above mentioned research articles that SMAT produces a refined grain structure which is induced by the rapid plastic deformation produced during SMAT process. This grain refinement is theorised to be formed due to the formation of DDW and DT. The surface roughness of the treated specimen also increased by $93 \%$ and the microhardness of the specimen increased with increasing time of SMAT and also with increasing ball diameters used in SMAT process. SMAT increases the wettability of the specimen.

Hydrogen embrittlement conclusively showed that the brittleness of the SMATed specimen increased when compared to the As Received Specimen. Corrosion resistance dropped with SMAT but annealing increased the corrosion resistance which is caused due to the diffusion of Chromium onto the surface of the metal from the matrix forming a more potent passive corrosion protective layer.

\section{REFERENCES}

[1] K.Lu, J.Lu, Nanostructured surface layer on metallic materials induced by surface mechanical attrition treatment, Materials Science and Engineering A 375-377 (2004) 38-45.

[2] Jian Lu, K.Lu, Generation of Nanostructure by SMAT basic concepts, different processes and applications, Alternative Processes, 163-170.
3] N.R.Tao Et. Al., An investigation of surface nanocrystallization mechanism in Fe induced by surface mechanical attrition treatment, Acta Materialia 50 (2002) 4603-4616.

[4] B.Arifvianto Et. Al., Effect of surface mechanical attrition treatment (SMAT) on microhardness, surface roughness and wettability of AISI 316L, Materials Chemistry and Physics 125 (2011) 418-426.

[5] T.Balusamy Et. Al., Effect of surface nanocrystallization on the corrosion behavior of AISI 409 stainless steel, Corrosion Science 52 (2010) 3826-3834.

[6] HAO Yun-Wei, et. al., Effect of SMAT on Corrosion behaviour of 316 SS, Journal of Iron and Steel Research International, 2009, 16(2), 68-72.

[7] Nairong Tao et. al., Development of nanostructures in metallic materials with low stacking fault energies during SMAT, Material Transactions, Vol. 44(10), 2003, 1919-1925. 